\title{
The Application of Electronic Map in Wireless Positioning System
}

\author{
Qinghui Wang, Wenzhou Wang, Dan Li,Lei Chang \\ College of Information Engineering, Shenyang University of Chemical Technology, Shenyang, 110142, China \\ E-mail: wangwenzhou130@126.com
}

\begin{abstract}
By using powerful secondary development tool MapXtreme and combining .NET development platform, Web Geographic Information System (WebGIS) used for the monitoring of the indoor personnel is designed. The system could display real-time location of the indoor personnel on the electronic map through getting the coordinates and other data measured by wireless positioning system combined with map matching. This system follows the browser/server mode (B/S mode), thus the visitors can easily access simply by the browser without the need of installing other application software.
\end{abstract} Map

Keywords-MapXtreme; WebGIS; Map Matching; Electronic

\section{INTRODUCTION}

With the rapid development of the Internet and geographic information technologies, there are increasing requirements for the Geographic Information System (GIS). Web Geographic Information System (WebGIS), as the juncture of web technology and GIS technology [1] [2], since its inception, its development is closely linked with the progress of computer technology. The purpose of this design is, in accordance with the demand of the development of electronic map navigation system, to investigate the current implementation techniques, discuss electronic map navigation system based on MapInfo platform and apply it to the specific system development practices.

The electronic map navigation system described in this paper is completed under multi-layer architecture of supporting .NET platform [3]. In this architecture, WebGIS system is constituted by the four large logical layer: WebGIS user interface layer (client), Web layer, WebGIS application service layer (middle layer), and data layer. System development process firstly designs system logic realization model based on object-oriented spatial data organization model and carrying out object-centered analysis, and then achieve the organization and packaging of system structure module by .NET organization means. Thus, on the one hand, the development speed of the prototype system could be improved; on the other hand, modularity and reusable extent of the system could be enhanced.

\section{THE OVERALL DESIGN OF THE SYSTEM}

The designed personnel monitoring system here is primarily used for the monitoring of indoor personnel. The system is mainly composed by wireless positioning system and Web Geographic Information System. Wireless positioning system here is not the focus of this study. Figure 1 is the overall design of the system.

Map workspace is made by the electronic maps drawn by the professional mapping software MapInfo Professional through MapXtreme Workspace Manager. Geographic Information System embeds MapXtreme for Windows, a COM technology-based control, in .NET development platform environment through COM component hosting technology as map application server [4], and the IIS is taken as a component of Web server. Wireless Positioning System stores positioning coordinates and other information in the database, and Web Geographic Information System obtains the needed coordinates and other information by connecting the database.

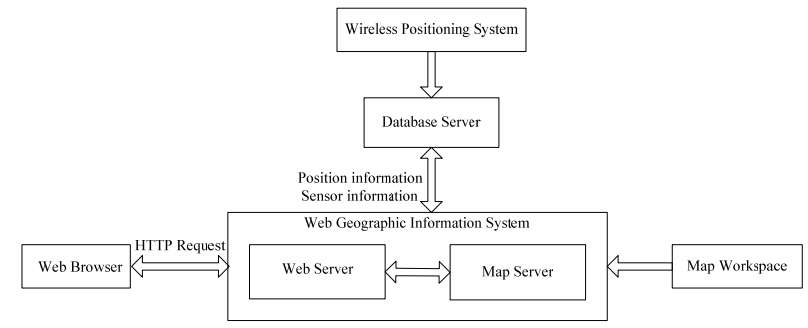

Figure 1. The overall design of the system.

\section{CONSTITUENT PART OF THE NAVIGATION SYSTEM}

\section{A. Map Workspace}

The laboratory internal map drawn in this design is drawn by professional mapping software MapInfo [5]. In order to improve the efficiency of the query and processing, and MapInfo carries out tiered map storage. Taking into account test the actual needs, the map is divided into three layers for storage, the three layers are respectively: the classroom, laboratory bench, and route. Figure 2 is the laboratory map drawn by the MapInfo software. Each MapInfo table is stored in each layer; each table is a set of MapInfo files, all MapInfo tables have the following files.

1) Attribute data's table structure file. TAB: Attribute data's table structure file defines the Map attribute data's table structure, including the number of fields, field names and field types and field width, index fields and the corresponding layers' critical spatial information descriptions. The table structure file is actually a text file can be opened in WordPad to see its contents. 
2) Attribute data file. DAT: Full map attribute data is stored in attribute data files. It is table structure description after the file header, followed by end-to-end specific attribute data record.

3) Cross-index file .ID: The cross-reference file records the position indicator of each spatial object in the map in spatial data file (. MAP), every four bytes constitute an indicator. The order of indicators is the same with the order of attribute data recording and store in attribute data file (.DAT). The cross-index file is actually a positioning table of spatial object.

4) Spatial data files. MAP: Specifically, it contains the spatial data of map object. Spatial data includes the geometry type of space object, coordinate information and color information. In addition, it also describes the record number of spatial object's corresponding attribute data records in the attribute data file (.DAT). In this way, when the user queries certain map object from the map, the associated attribute information can easily be found.

5) Index files .IND: The index file is not necessary, only when the users define index fields of the database, Maplnfo will automatically generate an index file. Each index field in index file has an index table. The total number of database records will be firstly given in each index table, and then according to the index sequence, the specific attribute data of each attribute data record in the corresponding index field and the record's record number in the properties file (.DAT) and cross-index index file (.ID).

The finished electronic map will be made into map workspace (.mws file) required by Web Geographic Information System via the Workspace Manager.

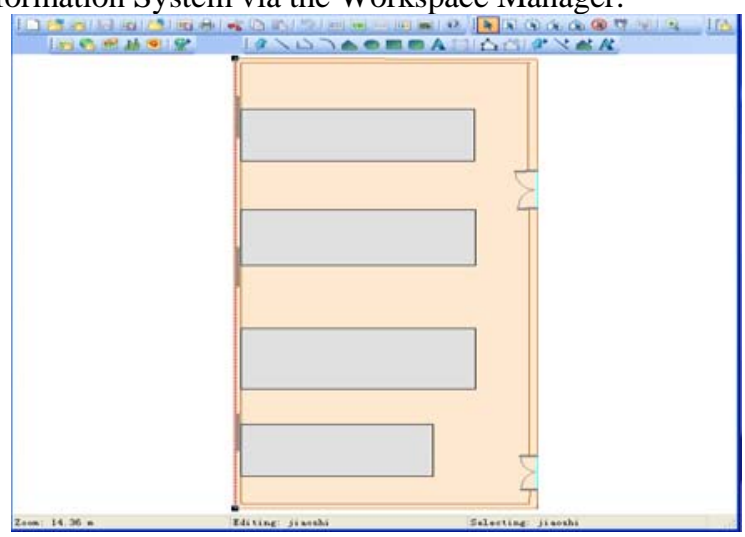

Figure 2. The laboratory map.

\section{B. The Access to Location and other Information}

The wireless positioning system gets a series of data about position through the sensor which will be processed in the background, and the obtained position coordinates and other data will be stored into the database. In order to obtain these data, it is necessary for Web Geographic Information System to connect to the database to get the required data. The database adopted in this design is oracle database, and
ADO.NET will be used for .NET platform to connect to the database.

The access to Oracle database requires not only the installation of Client software, but also the creation of a interception at Oracle Client for the access to server. The data source can be found in the tnsnames.ora file from the Oracle installation directory; the Oracle connection string and Oracle name resolution can't be separated. We define a database alias orcl in tnsnames.ora files as follows:

orcl=

$$
\text { (DESCRIPTION = }
$$

(ADDRESS_LIST =

$($ ADDRESS $=($ PROTOCOL $=$ TCP $)($ HOST $=$ 202.199.119.136) $(\mathrm{PORT}=1521))$

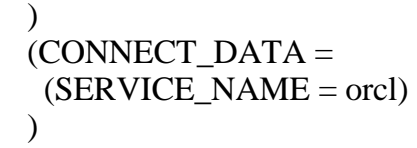

orcl alias defines Client's database address connection information, then database connection string can be defined as follows:

string oradb = "Data Source = orcl; User Id = fcs; Password = fcs;"; / / C \#

\section{Map Matching}

While moving the targets displayed on the electronic map, due to the positioning error of the moving targets and the electronic map's address matching error, it is possible to make that there is no staff displayed in the way. Map matching is a soft tech based positioning correction method, the basic idea of it is to link the moving targets' positioning track with the way network of the digital map, and thus the position of the moving targets relative to the map can be determined. By using the map matching method, with the use of prior information about the map data, the reliability and accuracy of the positioning system can be improved. Matching way choosing is the key for map matching, once matching way has been identified; only the projection of positioning point is needed, thus accurate map matching points can be obtained. This design adopts a comprehensive weight factorTWS as a basis of way choosing[6] as shown in formula (1), it take into account personnel and the closeness of the ways $\left(W S_{D}+W S_{P}\right)$ as well as the consistent level between the heading of the moving personnel and the direction of the way $\left(W S_{H}\right)$.

$$
T W S=W S_{H}+\left(W S_{D}+W S_{P}\right)
$$

The largest way of comprehensive weight factor TWS is the optimal matching way. Direct projection of the current anchor point to the way is the accurate matching point [7] [8].

The calculation method of each weight value factor is described as below:

1) The consistent level between the heading of the moving personnel and the direction of the way: As shown in 
figure $3, P_{1}, P_{2}, P_{3}$ are the anchor points which the moving personnel acquired in the sequential order last time and $P_{4}$ is the current anchor point. When the moving person leave off the road 1 , the optional matching road include road 2,3 , and 4. Here, $\beta$ is defined as the heading angle (which is the angle between the movement direction and the north direction) of the current moving person. $\beta^{\prime}$ is defined as the angle between the direction of the road and the north direction. As shown in figure 3, the direction of the road 2, 3 and 4 is $0^{\circ}, 90^{\circ}$ and $180^{\circ}$. If the value of $W S_{H}$ is larger of a road, the closer between the heading of the moving personnel and the direction of the way.

$$
W S_{H}=A_{H} \cos \left(\Delta \beta^{\prime}\right)
$$

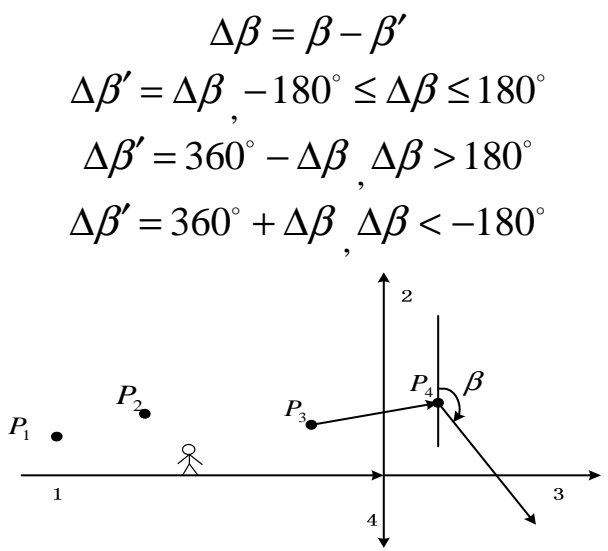

Figure 3. The consistent level between the heading of the moving personnel and the direction of the way.

2) The closeness between the moving personnel and the ways: From the distance and angle, two weight factor $\left(W S_{D}\right.$ and $W S_{P}$ ) are constructed to reflect the closeness between the moving personnel and the road accurately.

As shown in figure 4, $\mathrm{P}$ is the current anchor point. $\mathrm{AB}$ is the road and $C$ is the matching point of $P$.

The distance between the point $\mathrm{P}$ and road $\mathrm{AB}$ :

$$
D=\frac{x_{3}\left(y_{1}-y_{2}\right)-y_{3}\left(x_{1}-x_{2}\right)+\left(x_{1} y_{2}-x_{2} y_{1}\right)}{\sqrt{\left(x_{1}-x_{2}\right)^{2}+\left(y_{1}-y_{2}\right)^{2}}}
$$

$W S_{D}=A_{D} / D$ The higher of the value, the closer between the anchor point and the object road

As shown in figure 5, C is the intersection point of the road. $A B$ is the road and $C$ is the matching point of $P . P$ is the current anchor point and $\alpha$ represents the angle from a road to $\mathrm{CP}$.

$W S_{P}=A_{P} \cos (\alpha)$ The higher of the value, the closer between the anchor point and the object road

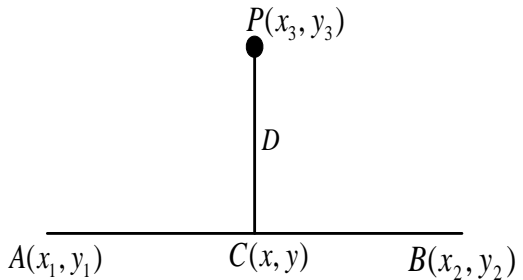

Figure 4. The distance between the personnel and the road.

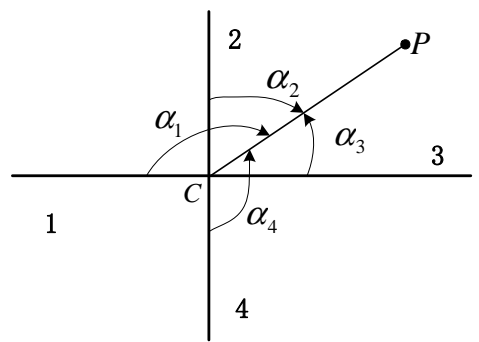

Figure 5. The angle between the personnel and the road.

Because comprehensive matching factor TWS give full consideration to the spatial relationship of moving personnel location, personnel movement direction, way, and intersection, the matching algorithm has higher accuracy and adaptability compared to traditional matching methods relying on distance or direction. Especially when the positioning error is relatively large, it's still able to correctly identify the way of moving personnel and ahs high antiinterference ability. Figure 6 is a map matching algorithm process.

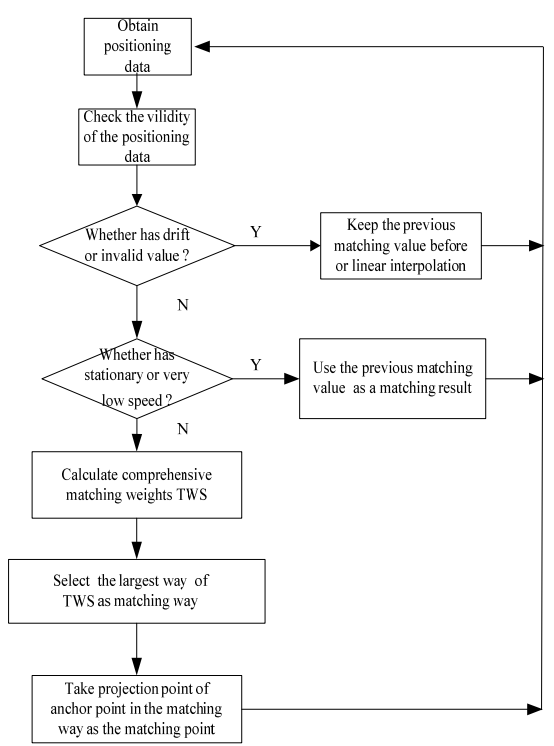

Figure 6. The map matching algorithm process. 


\section{SYSTEM WORKFLOW}

The User carries on the corresponding map operation at Client. Client browser makes an HTTP request to the Web Server and submits the Form simultaneously. ASP application determines the user's map operation request type according to the information about the submitted form and accesses the MapXtreme Map application server's Map engine. After making corresponding spatial analysis and processing, Map engine will pass the result to the Web Server in the form of bitmap (. Gif, Jpg, Png, etc.), Web Server embeds this bitmap file in an HTML page and then returns to the Client browser. The real-time positioning of personnel is the most important part of the system; Figure 7 is flow chart for the real-time positioning work of personnel.

The codes of coordinates obtained from the database are as follow:

string strSql ="SELECT * FROM ( "+"select C_SBBH,to_char(D_SJ,'yyyy-mm-ddhh:mm:ss'), N_TD, N_XT, N_YQL, N_XPOS, N_YPOS ,N_ZT, N_HPOS,"+ " ROW_NUMBER() OVER(PARTITION BY R.C_SBBH ORDER BY R.D_SJ desc) AS emp_id "+" from FC_RECORD R where D_SJ > sysdate - 5/(24*60) "+ " ) WHERE emp_id=1";

Wherein, field N_XPOS is $\mathrm{X}$ coordinate and field N_YPOS is Y coordinate.

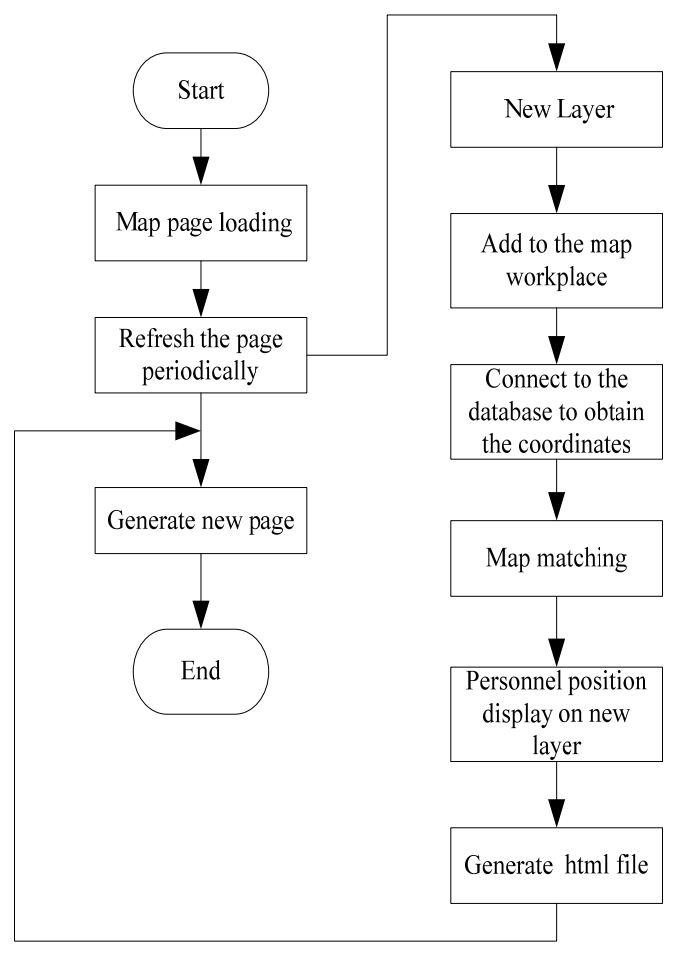

Figure 7. The flow chart of positioning work.

\section{TEST RESULTS AND CONCLUSIONS}

A large laboratory in school's No. 6 laboratory building is taken as the test site to test the system. The laboratory is 15 meters long and 8 meters wide, and the designed personnel monitoring system is tested after wireless positioning system in the laboratory is finished. Figure 8 is the system test chart.

The test results show that this system could make accurate indoor personnel position monitoring through the wireless positioning system. The map-matching algorithm will conduct effect correction when there is an error in the coordinates provided by wireless positioning system.

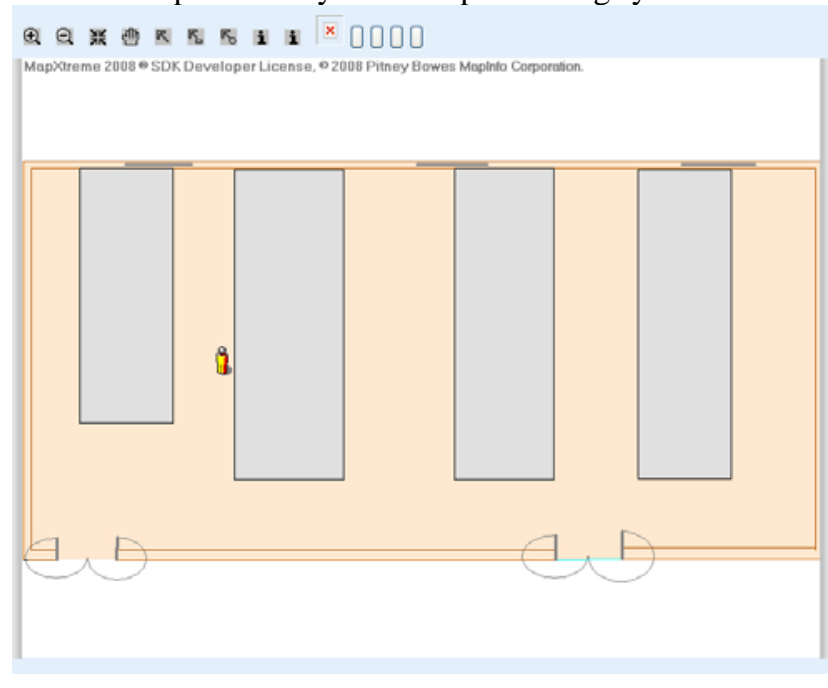

Figure 8. The system test chart.

\section{REFERENCES}

[1] J.F. Liu, F.P. Zhang, X.Ch. Yang, WebGIS and its application. Jiangxi Meteorological Science and Technology, l (l):16-17.

[2] G. Wang, H.B.Lu, S.H. Yan, GSI's development direction- WebGIS, Northeast Surveying and Mapping, Volume 24, No. 4 of 2003,13-16.

[3] G.R. Li, WebGIS's three-layer application architecture model and its implementation techniques. Journal of Southern metallurgy college 2003, 24 (1):12-15.

[4] N. Liu, R.Y. Liu, Distributed WebGIS framework and implementation methods based on $\mathrm{COM}+$. Journal of Image and Graphics, 2004, 9 (1):99-104.

[5] W.Q. Luo, Y. Luo, K. Zeng, The construction of digitized Geographic Information System and advanced applications of MapInfo. Tsinghua University Press, 2003.4:125-204.

[6] J.Y. Chen, G.R.Xiao, Weight-based map matching algorithm. Computer Engineering and Applications, 2005, 41 (11): 168 - 170.

[7] H.L. Zhong, R.H. Tong, J. Li, GPS positioning and map matching method. Mini-Micro Systems, 2003, 24 (1): 109-113.

[8] N. Wang, Y.F. Wang, J.R. Liu, A point-based map matching algorithm .Journal of Northeastern University 1999, 20(4): 344 - 347. 